\title{
690 TIGIT BLOCKADE IMPROVES ANTI-TUMOR ACTIVITY OF EX VIVO EXPANDED NK CELLS
}

Md Faqrul Hasan*, Alicja Copik. University of Central Florida, Orlando, FL, United States

Background Natural killer (NK) cells are innate immune cells that directly kill and coordinate responses against cancer prompting interest in using ex vivo expanded NK cells as an adoptive cell therapy for treatment of cancer. NK cells express a set of activating and inhibitory receptors that regulate their activity. Inhibitory receptor TIGIT ( $\mathrm{T}$ cell Immunoreceptor with Ig and ITIM domain) is upregulated on intratumoral NK cells in some cancers, inhibits NK cell activity and promotes NK cell exhaustion. In this study, the effect of TIGIT blockade on the anti-tumor activities of ex vivo expanded NK cells was evaluated.

Methods NK cells were activated overnight with cytokines or ex vivo expanded with PM21-particles. Their TIGIT expression was determined with qRT-PCR and flow cytometry. Cytotoxicity was assessed by kinetic, imaging-based assay (Incucyte S3) against A549 and NCI-H1299 cells cultured in 3D. Cytotoxicity was calculated based on untreated controls at different time-points. Results from multiple donors were normalized to cytotoxicity of NK cells with isotype for individual donors and was compared to the cytotoxicity of NK cells with antiTIGIT. Unpaired t test was used to determine statistical significance. K562 cells stably expressing Polio Virus Receptor (PVR), were used to restimulate A549 spheroid-exposed NK cells to measure IFN $\gamma, \mathrm{TNF} \alpha$ and degranulation. Furthermore, phenotypic changes of NK cells upon TIGIT blockade were examined by analyzing a set of activating and inhibitory receptors by flow cytometry.

Results The effect of NK cell expansion/activation on TIGIT expression was assessed. TIGIT was upregulated on expanded and cytokine-activated NK cells both on mRNA and protein level. The effect of TIGIT blockade on NK cell cytotoxicity was examined by co-culturing PM21-NK cells with cancer cells in the presence of anti-TIGIT antibodies or respective isotypes. TIGIT blockade significantly increased cytotoxicity of PM21-NK cells against A549 (1.3 fold, P $<0.0001)$ and NCI-H1299 (1.3 fold, $\mathrm{P}=0.0003)$ spheroids after 48 h. To access exhaustion, NK cells exposed to A549 spheroids for 7 days were restimulated with $\mathrm{PVR}^{+}$K562 cells. TIGIT blockade prevented NK cell exhaustion resulting in increased expression of IFN $\gamma, \mathrm{TNF} \alpha$ and surface CD107a on restimulated NK cells. TIGIT blockade did not result in changes to the surface phenotype of NK cells.

Conclusions TIGIT was highly expressed on expanded and cytokine-activated NK cells. TIGIT blockade improved antitumor activities of PM21-NK cells. Thus, PM21-NK cells and TIGIT antibodies have translational potential as a combination therapy to improve anti-tumor response.

http://dx.doi.org/10.1136/jitc-2021-SITC2021.690 